\title{
Localization of Suborbital Vehicle Using On-Board Map and Camera Image
}

\author{
M. Jisha \\ M.Tech Robotics, Dept. of Mechanical Engineering, SRM University, Chennai, India \\ Special Research Student, Dept. of Mechanical and Control Engineering, Kyushu Institute of Technology, Japan
}

\begin{abstract}
Recently, unmanned aerial exploration of planets is an emerging field of interest for researchers all over the world. One of the major challenges that lie in the exploration aircraft is its localization in an environment where there is no useful navigation system like GPS. Although several localization algorithms were proposed, like SURF and SIFT, they suffer from premature convergence in an unknown and harsh terrain atmosphere (using satellite image mapping). The aim of this research is to develop a localization algorithm to detect the optimum features from the video, obtained onboard, and performs fast feature mapping of the features with the satellite image. The features are then mapped to the exact location on the database image and the path of the kite plane is obtained on the database image using RANSAC. The localization algorithm was evaluated in flight experiments using a kite plane. The experimental result of the algorithm showed localization of the aircraft using OpenCV (using CPP Console). The experimental results showed that the proposed algorithm (LAIGDE) outperforms the traditional mapping techniques like SURF with high accuracy and robustness.
\end{abstract}

Keywords: SURF, RANSAC, Histogram, GPS, Homography, Mapping.

\section{INTRODUCTION}

\section{A. Image Matching Using Feature Points}

Mapping, of the exact image, and feature detections are major areas of research in computer applications related to autonomous vision-based localization and classification. Feature detection is the process where we automatically examine an image to extract features that are unique to the objects in the image, in such a manner that we are able to detect an object based on its features in different images [1][2][4]. This detection should ideally be possible even if the is taken with different transformations, i.e. scale and rotation, or when parts of the object are occluded [7][18]. The image matching processes can be divided into 3 overall steps:

Feature detection: In this step the distinctive interest points are selected from the whole image (cornersjunctions and high-frequency blobs). The feature detector should be repeatable such that the same feature point can be obtained under different viewing conditions any number of times with high accuracy.

Feature description: A feature descriptor represents the neighborhood of every interest point by a feature vector. An idle feature descriptor must be distinctive, robust to noise and must be invariant to displacement, geometry and photometric deformation (i.e. they must provide an additional description about the detected features/key points from the detector) [15][18][20]. A high-dimensional feature descriptor is more distinctive but consumes more time compared to a less dimensional descriptor.

Feature mapping: During feature mapping the matching of the descriptor vectors between the two compared images is carried out efficiently [7]. The matching is based on the Euclidean distance. Hence, a tree structure is created and a proper search method is utilized for mapping [5][16].

\section{B. Application of Image Mapping}

The application of image based localization algorithm is in space missions and satellite-based research on the investigation of change in Earth's landscape. During space missions where no GPS values are available, the image base mapping helps in providing the current location. The space-based application of localization of the image mapping was introduced by JAXA, Japan for its unmanned spacecraft as shown in Fig.1 (a) (Launched on 9 May 2003) to return a sample of materials from a small near-Earth asteroid named Itokawa. After arriving at the asteroid (Itokawa), the spacecraft (Hayabusa) studied the asteroid's shape, spin, topography, colour, composition, density, and history.

The most recent research involves the Mars rover Localization as shown in Fig.1 (b) for JAXA's rover named MELOS-1 (to be launched by 2020 or 2022, Landing date 2021 or 2023) for the investigation of the evolution of Martian atmosphere, i.e. the water and climate of Mars.

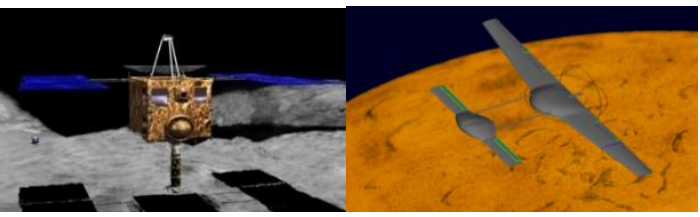

(a)

(b)

Fig.1. (a) Spacecraft Hayabusa near the surface of Itokawa (b) MELOS-1 over Mars Surface

Rest of the paper is organized as follows: Section 2, includes the discussion of the related works in image mapping and localization approaches. The proposed 
algorithm for image mapping and localization of the suborbital vehicle in a GPS-denied environment is discussed in section 3 . We discusses the experiments used for validation of our proposed algorithm (LAIGDE) and collated results in section 4 . Section 5, concludes the work and highlights about the area of advancements in this proposed approach.

\section{LITERATURE REVIEW}

The literature survey is done to obtain the foregoing details and methodology of the mapping algorithm and to gain the knowledge of algorithm for target detection, the survey on vision system had been carried out. Chandrakala et al. [1], studied the collection of earth data and creating a map like an image by classifying the satellite image into 3 different regions water, urban and Greenland. At first, the obtained satellite image is pre-processed and then using the SURF algorithm the features are detected and described (to make the algorithm scale and Orientation invariant). The bag of features is applied to the LDA and a threshold based comparison is carried out for the classification of the topography. Frederic et al. [2], proposed position estimation for a mobile robot using vision and odometry. This algorithm helped in locating a mobile robot in an unknown environment by combining the position estimation from odometry and the visionbased estimation of the environment using a camera. A database based fixed objects were used as the landmark. The system calculates the angle of orientation and corrects the robot as soon as an error occurs in the angle with respect to the landmark.

Luo et al. [4], compares the performance of SIFT, PCSSIFT, and SURF based on the times taken for feature detection and the number of features detected. It was found that the SIFT is more accurate but consumes more computation time and is less robust. Whereas SURF is robust with less time taken in detecting features, which in turn is less in number compared to the features detected by SIFT. Here K-nearest Neighbor is used in detecting the correct keypoint matching for robustness. Chao et al. [5], proposed a location estimation algorithm for a UAV in a GPS-denied environment where they employed SIFT for feature detection and used Google image as a database image. The output of SIFT and the IMU module is used in a real-time environment to determine the position of the UAV. Here 3D based SLAM map of the location of motion of the UAV is created. But the drawback of the algorithm lies in the computational cost with the increase in number of features being detected.

Mark et al. [8], proposed FAB-MAP to overcome the difficulty of detecting closure in matrices using slam algorithm. They made use of Chow-Liu based mapping where a probability-based matching is carried out. The database contains keypoints with probabilistic relations as their weight .Their weight functions were used for future mapping. Jan et al. [11], developed a K-D tree based for shape registration based on the data obtained from the scanning of the 3D object. Each shape features are stored as a tree structure for easy retrieval and identification of the object compared to an iterative closest point algorithm.
Glanpaolo et al. [13], proposed a vision based navigation architecture which combines inertial sensors, Visual odometry, and registration of the onboard video to a georeferenced aerial image. The vision-based navigation system is capable of providing an estimate of the location of the UAV even without the GPS system.

Xing et al. [14], studied a hessian based algorithm for feature detectors and a circle shaped filter is used instead of a square shaped filter for increasing the efficiency when to compare to SURF algorithm. It eliminates false matching and enhances the robustness compared to SURF. Rochdi et al. [15], developed an extension of SURF as SURF-X which makes use of the HSV (Hue, Saturation, Value) and SURF-HISTEQ for invariance to rotation and invariance to scale respectively. SURF-X is proposed to be more efficient in feature description compared to SURF algorithm.

From the literature studied it can be concluded that in the field of autonomous navigation a mapping algorithm for localization must be robust and invariant to orientation and illumination changes of the localization. As per the study, SIFT mapping algorithm is less robust when viewed in a dynamic environment compared to SURF algorithm. SLAM provides a probabilistic approach, hence, its computation cost is very high compared to SIFT and hence this makes SLAM inefficient for a dynamic localization of suborbital vehicle. SURF on other hand has less computation cost and tends to be robust in a dynamic environment. But the major disadvantage of SURF lies in the mapping mismatches due to its filters. The objective which is derived from the various literature papers includes autonomous localization of a suborbital vehicle, hence developing a mapping algorithm which would be invariant to scale changes, illumination and orientation changes. Hence, this robust matching algorithm can be employed in a GPS-denied environment for its autonomous localization compared to traditional SURF approach. In this work we have propose the following:

- To design a fast location mapping algorithm which is illumination invariant, Orientation invariant and scale invariant.

- To localize suborbital vehicle in a GPS-denied environment where the location of the suborbital vehicle is determined only using our proposed approach.

- To create a localization algorithm that outperforms the existing SURF and SIFTS approach.

\section{PROPOSED SOLUTION}

\section{A. Matching Algorithm}

The objective of this research work as stated before is to develop a robust image mapping algorithm for navigation of a sub-orbital vehicle in a GPS-denied environment. The major objectives of the project lie in the localization in an environment where there is no availability of GPS data. The localization algorithm to be implemented for the vehicle should be invariant to its orientation, speed, height and the nearby illumination changes. Hence, a more robust 
INTERNATIONAL JOURNAL OF INNOVATIVE RESEARCH IN ELECTRICAL, ELECTRONICS, INSTRUMENTATION AND CONTROL ENGINEERING Vol. 4, Issue 5, May 2016

matching algorithm is to be developed for a dynamic and an unknown environment.

To find the best match between two images and to localize the vehicle in a GPS-denied environment, the following steps are carried out:

Contrast enhancement of the images: Using Histogram Equalization

Feature detection: Using Hessian matrix

Feature description: Using Haar wavelet

Feature mapping: Using Euclidean distance

Path formulation: Using RANSAC

- Contrast enhancement of the images : Histogram Equalization

Histogram equalization is a method widely used in image processing for contrast adjustment using the image's histogram. The luminous intensity can be better distributed on the histogram. This allows for areas of lower local contrast to gain a higher contrast. Histogram equalization accomplishes this by effectively spreading out the most frequent intensity values. In applying histogram equalization (as shown in Fig. 2) on an RGB image, the image is first converted to another colour space, lab colour space, or HSL/HSV in particular, and then the algorithm can be applied to the luminance or value channel without resulting in changes to the hue and saturation of the image and again convert it to RGB colour space.

- Feature Detection: Using Hessian Matrix

For a given point $(X, Y)$ in an image $I$, the Hessian matrix $H(X, \sigma)$ in $X$ at the scale $\sigma$ is defined as follows in Eq. (1):

$$
H(X, Y, s)=\left[\begin{array}{ll}
H_{X X} & H_{X Y} \\
H_{X Y} & H_{Y Y}
\end{array}\right]
$$

Where $H_{x x}(\boldsymbol{x}, \sigma)$ is the convolution of the Gaussian second order derivative $\frac{\partial^{2}}{\partial x^{2}} g(\sigma)\left(G_{X X}\right)$ with the image $I$ at the point $x$ (similarly for $H_{x y}(\boldsymbol{x}, \sigma)$ and $H_{y y}(\boldsymbol{x}, \sigma)$ ).
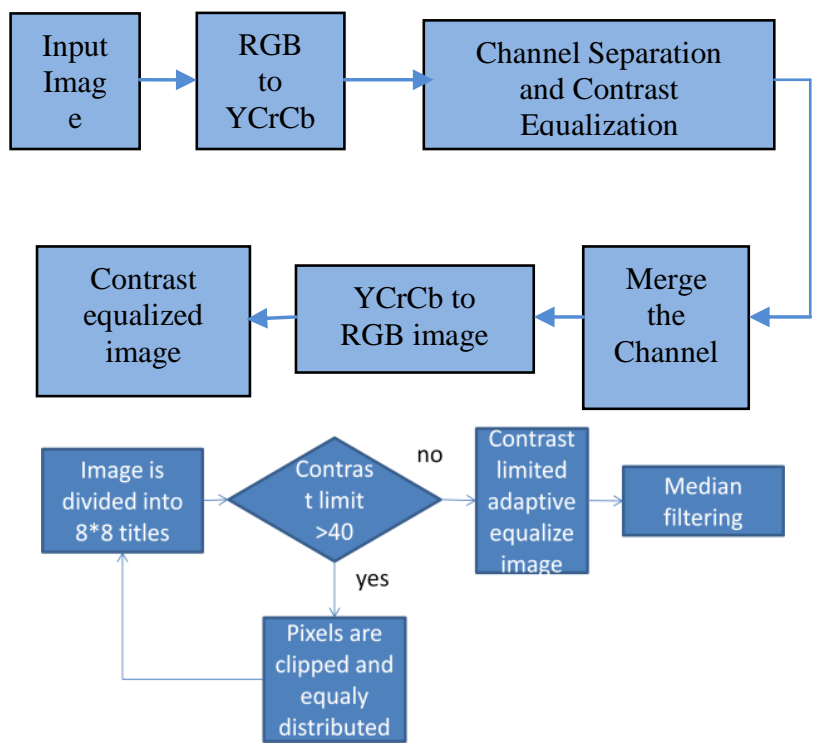

Fig. 2. Contrast Enhancement of the Image

$H_{X X}=G_{X X} \times I(X, Y), H_{X Y}=G_{X Y} \times I(X, Y), H_{Y Y}=G_{Y Y} \times I(X, Y)$
$G_{X X}=\frac{\partial^{2} g(\sigma)}{\partial y^{2}}=G_{Y Y} ; \frac{\partial^{2} g(\sigma)}{\partial x \partial y}=G_{X Y} ; \frac{\partial^{2} g(\sigma)}{\partial x^{2}}=G_{X X}$
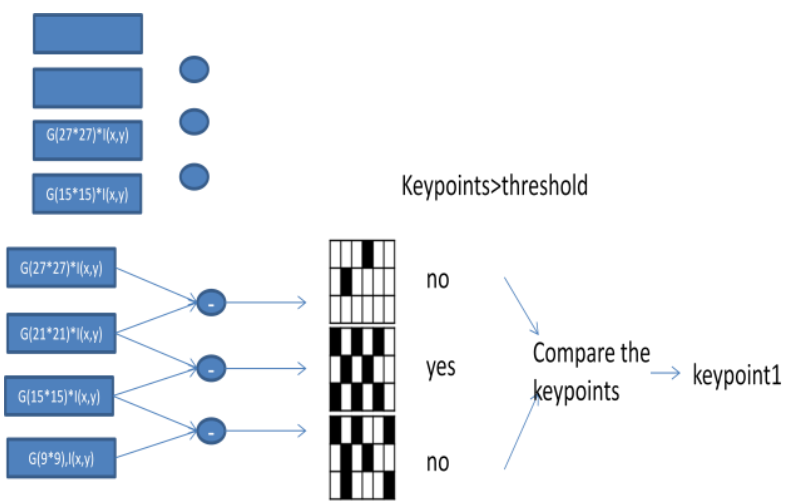

Fig. 3.Hessian based keypoint detection

Hessian Threshold is detected using Eq. (3)

HessianThreshold $=G_{X X} G_{Y Y}-\left(a G_{X Y}\right)^{2}$

In this research, the Hessian threshold value is set to 6000 . Hence, for any frame if the detected Hessian Threshold value is greater than or equal to 6000 only then the preceding procedures are carried out on the image to find the best match.

\section{- Feature Description: Using Haar Wavelet}

In this research project, Haar Wavelet is used to make the algorithm invariant to image rotation and orientation. The rotation/orientation of the interest point is estimated using the Eq. (4). The Haar sequence was proposed in 1909 by Alfréd Haar.

$\theta=\tan ^{-1}\left(\frac{\partial y}{\partial x}\right)$

Since modern cameras are capable of capturing images of higher resolution, it is highly required to compress the image before storage or transfer from one location to another location. In such situations, Haar transform can be used for image compression.

- Feature Mapping: Euclidean Distance

From the detected features a $k$ - $d$ search tree is formed based on the frame number and time

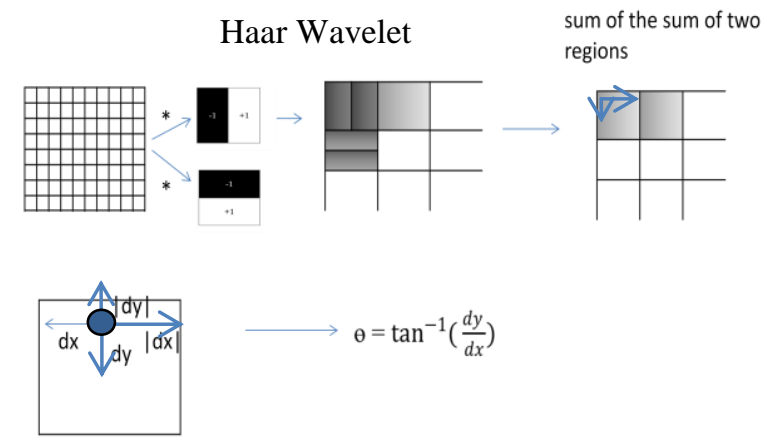

Fig.4. Orientation detection of the interest point 
INTERNATIONAL JOURNAL OF INNOVATIVE RESEARCH IN ELECTRICAL, ELECTRONICS, INSTRUMENTATION AND CONTROL ENGINEERING Vol. 4, Issue 5, May 2016

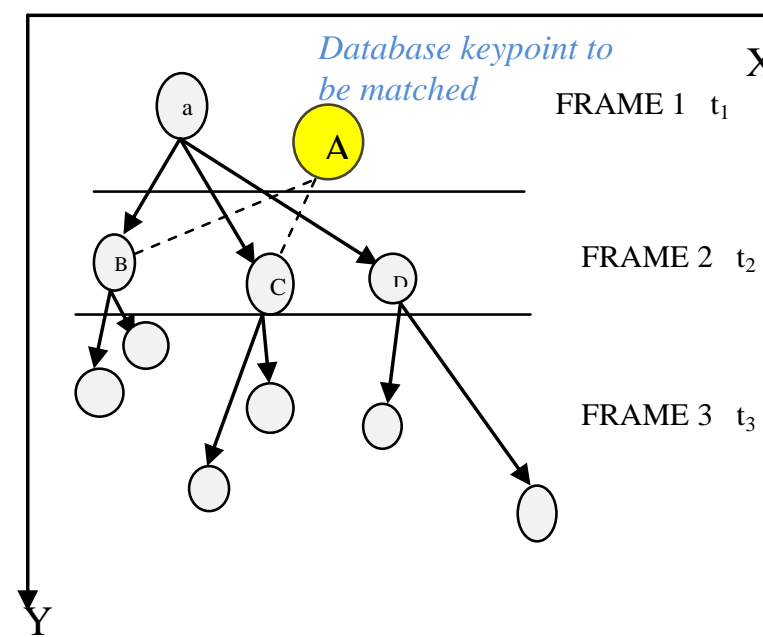

Fig. 5. Feature matching using $k-d$ search tree

From the above Fig. 5 the node in 'yellow' represents the obtained keypoint in the database image compared to the 'grey' nodes which resemble the keypoints obtained from the panorama image (obtained by stitching video frames).

\section{- Path Formulation: RANSAC}

Using RANSAC inliers are obtained (best suitable path is formulated) from the detected machining points using probabilistic approach.

$N=\frac{\text { Number of inliers in data }}{\text { Number of points in data }}$

A common case is that $N$ is not well known beforehand, but some rough value can be given. Assuming that the ' $n$ ' points needed for estimating a model are selected independently, $N_{n}$ is the probability that all $\mathrm{n}$ points are inliers and $1-N_{n}$ is the probability that at least one of the ' $n$ ' points is an outlier, a case which implies that a bad model will be estimated from this point set. That probability to the power of $k$ is the probability that the algorithm never selects a set of ' $n$ ' points which all are inliers and this must be the same as $(1-P)$. Consequently, $1-P=\left(1-N^{n}\right)^{k}$

where,

$k=\log (1-p) / \log \left(1-N^{n}\right)$

To gain additional confidence, the standard deviation or multiples thereof can be added to $k$. The standard deviation of $k$ is defined as follows:

$S D(k)=\frac{\sqrt{1-N^{n}}}{N^{n}}$

Match finding
if $((\mathrm{AB} / \mathrm{AC})<3)$;
//Euclidean distance 3 is set as threshold
then $\mathrm{ACCEPT}$ ' $\mathrm{B}$ ' as the closest node
else
if $((\mathrm{AC} / \mathrm{AB})<3)$;
Then $\mathrm{ACCEPT} \mathrm{C}$ ' as the closest node
Else
If $(\ldots .$.
$\ldots .$.
Else
REJECT 'B'
//node b is not the closest node compared to node c

\section{Algorithm 1: LAIGDE algorithm}

Load Database image;

Load Convert Video to panorama image;

$\operatorname{For}(\mathrm{i}=0 ; \mathrm{i}<1000 ; 1++)$

Convert_YCrCb2BGR(Hist_equ(convert_BGR2YCrC

$\mathrm{b}($ database image $))=[\mathrm{a}]$;

Convert_YCrCb2BGR(Hist_equ(convert_BGR2YCrC

$\mathrm{b}($ panorama image $)))=[\mathrm{b}]$;

If $([\mathrm{a}] \|[\mathrm{b}])=$ ! 0)

Then

intminhessian $=6000$;

detect(keypoint_[a]);

detect(keypoint_[b]);

descriptor_a=extract(keypoint_[a]);

descriptor_b=extract(keypoint_[b]);

Create kd_tree[descriptor_a];

Create kd_tree[descriptor_b]

match(descriptor_a, descriptor_b);

distance(discrptor_a, descriptor_b);

Set min_dist $=1$;

Set max_dist $=100$;

If(distance $<3 *$ min_dist)

Then

Cout $<<$ "good match";

Draw(match);

Else

Cout $<<$ "no match";

Homography([a],[b],10);

ElseCout $<<$ "sorry couldn't load the image";

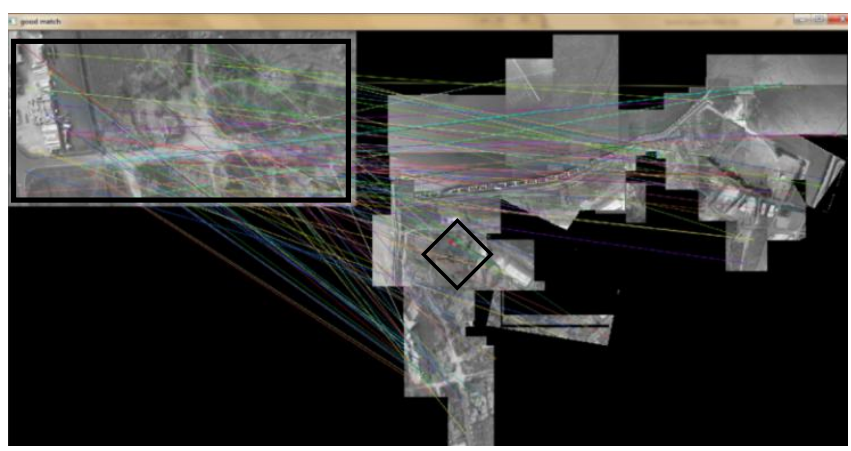

Fig. 7.Matching of the two images using the proposed approach

Fig. 6 and 7 represent the LAIGDE matching algorithm for localization of a vehicle in a GPS-denied environment with high accuracy and robustness.(i.e. fewer mismatches)

\section{B. Experimental Setup: Kite plane test}

A kite plane (captive plane) is a small flying machine that is controlled remotely by an operator on the ground using a hand-held radio transmitter. The receiver within the craft sends signals to servomechanism, which moves the control surface, and also communicates with the ground based transmitter, this in turn, affects the orientation of the plane. There are multiple applications of kite plane, such as scientific, government and military organization for gathering weather readings, aerodynamic modeling and testing and even using them as drone or spy planes. A Kite plane of $2.2 \mathrm{~m}$ was operated using ground-based control. 
INTERNATIONAL JOURNAL OF INNOVATIVE RESEARCH IN ELECTRICAL, ELECTRONICS, INSTRUMENTATION AND CONTROL ENGINEERING Vol. 4, Issue 5, May 2016

The kite plane was used as a mean to obtain the video for this project and also contained other research components of my lab as shown in Fig. 9.

Table 1: Specification of the Kite Plane

\begin{tabular}{|l|l|l|}
\hline Parameter & Unit & value \\
\hline Total length & $\mathrm{mm}$ & 2280 \\
\hline Span & $\mathrm{mm}$ & 2780 \\
\hline Height & $\mathrm{mm}$ & 1130 \\
\hline Weight & $\mathrm{kg}$ & 18 \\
\hline Max. Velocity & $\mathrm{m} / \mathrm{s}$ & 20 \\
\hline Max. Altitude & $\mathrm{m}$ & 200 \\
\hline
\end{tabular}

A black Sony handy-cam HRD-XR550E was mounted on the kite plane in Z-direction (facing the ground) for fetching the video of the place. The kite plane with the camera mounted on it with all the other components is as shown below in Fig. 8 (a) and (b).

From the video obtained from the kite plane, a large variation in illumination was clearly seen. From a research project of the same lab, GPS-based localization map of the kite plane was obtained. The major disadvantage of GPS data lies in its discontinuity. Various trials were carried out on the on-board GPS sensor to transmit values to the ground station which is as shown below in Fig. 10.

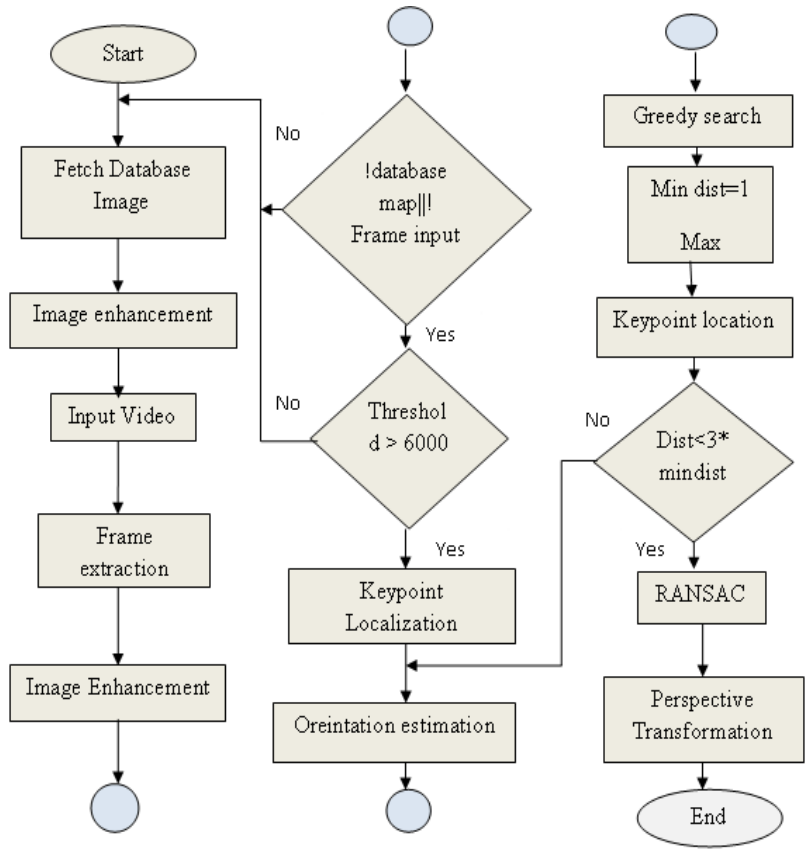

Fig. 6.LAIGDE Matching Algorithm

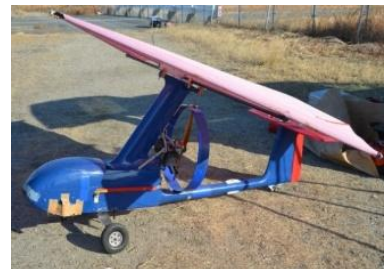

(a)

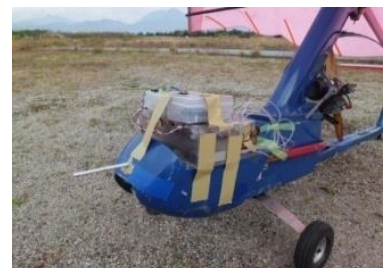

(b)
Fig. 8. Flight test of (a) Kite plane with camera mounted (b) Kite plane with all the experiments

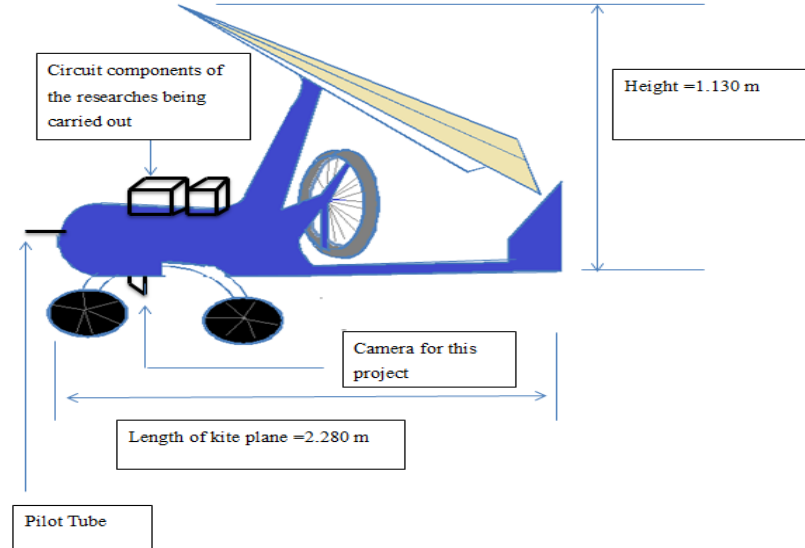

Fig 9. Schematic diagram of kite plane

The output obtained from the camera for localization of the Kite plane is as in Fig. 11. The Google map image of the place is as shown in Fig. 12 (a).

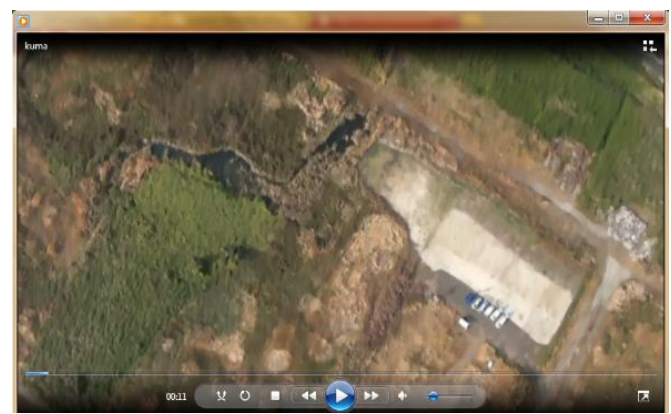

Fig. 10. Video frame obtained from the kite plane

The panorama view of the location was obtained after image stitching from the obtained video from the Kite plane using Microsoft research Composite Editor as in Fig. 12 (b), to obtain a panorama as shown in Fig. 13.

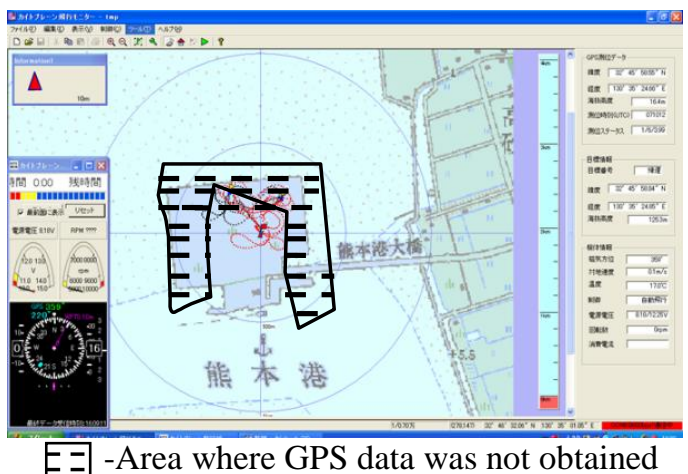

Fig. 11. GPS Data Obtained

This stitched image can be used as the database image if the map of the environment is not previously known. Hence, using this panorama image the present location of the kite plane any given time can be obtained periodically. 


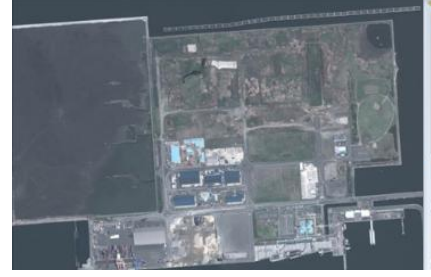

(a)

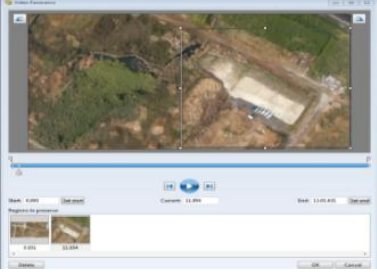

(b)
Fig. 12. (a) Kumamoto port Google Image Figure (b) panorama creation using Microsoft Composite Editor

Image stitching helps in combining the multiple frames with overlapping fields of view to produce a segmented panorama or higher-resolution image. The Panorama image from video obtained from the kite plane test would be considered as the database image to match it with the video obtained from the successive kite plane test to localize the plane.

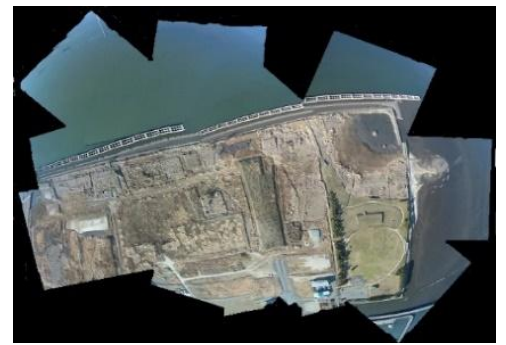

Fig. 13. Panorama image obtained

The proposed algorithm maps the panorama image obtained from the video. (as obtained in section 2)

\section{RESULT}

The input video from the Kite plane test was used in the mapping of its location on the database image (Google image). Here the Hessian threshold value was set to 6000 with contrast adjustment and the Euclidian mapping threshold less than 2 as shown in Fig.14.

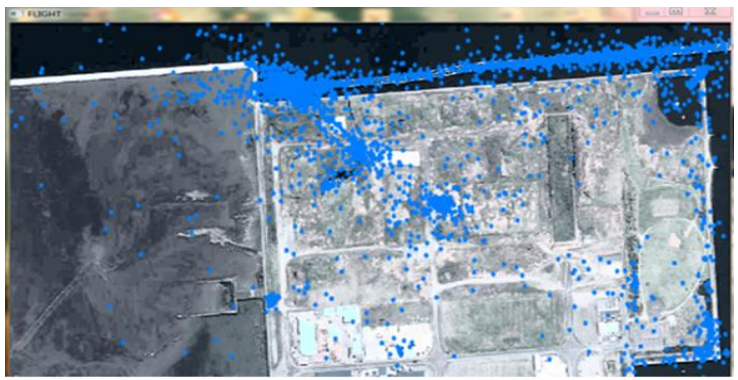

Fig. 14. Matching output for localization

- Error Analysis

For varying threshold values the localization points obtained varied. Hence, a different value of threshold values was applied to obtain the most desired output of the path taken by the kite plane.

- Selecting the Desired Hessian Threshold Value for SURF

For the minhessian threshold at 400 and 4000, more error prone,less matching points for localization were obtained

as shown in Fig.15 and 16. A fair matching was obtained at the minhessian of 6000 .

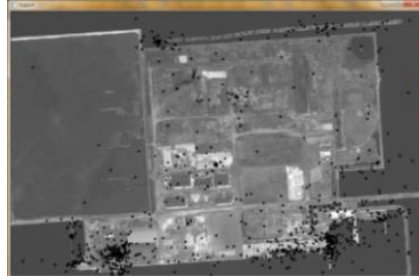

Fig.15Image matching with Hessianthreshold $=4000$

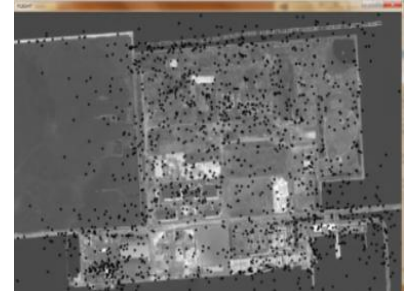

Fig16Image matchingwith Hessianthreshold $=400$

At a greater value of the HessianThreshold, the frame of image from the video input is considered only when the number of features is above the threshold value and hence it shows less mismatched and scattered points compared to the lesser value of HessianThreshold as shown in Fig. 17 and 18 .

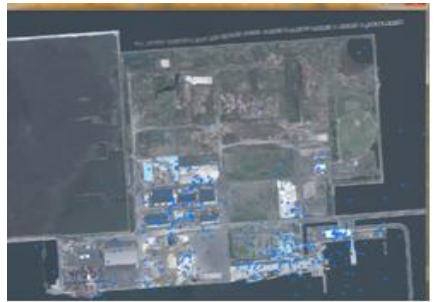

Fig. 17. Image matching at Hessianthreshold $=400$

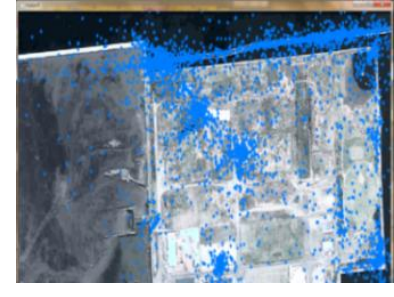

Fig. 18. Localization at Hessian threshold $=6000$

\section{- Providing a More Strict RANSAC Condition}

Setting the distance vector at a lower value (i.e setting the minimum matching distance between the vectors (of keypoints) more than or equal to a small integer value (i.e 2 or 3) would help in rejecting the less mismatched points. The RansacReproj Threshold is the maximum reprojection error allowed to treat the localized keypoint location as inliers value.

\section{||dstPoints ${ }_{i}$-convertPointsHomogeneous $\left(\right.$ HsrcPoints $\left._{i}\right) \|>$ ransacReprojThreshold}

When this condition is fulfilled the keypoints localized are rejected. In my experiment, this value is set to 10 .

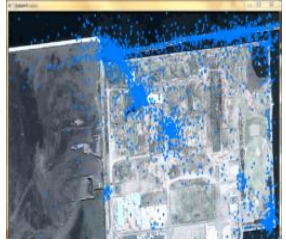

Hessain-

threshold $=6000$,

Ransacme $=5$

Dist $<2 *$ min dis

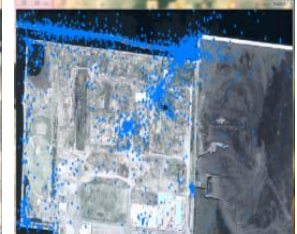

Hessainthreshold $=6000$,

Ransacme $=10$

Dist $<2 *$ min dist

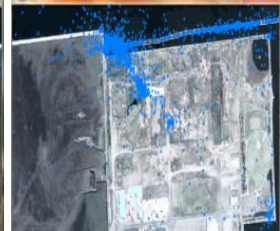

Ransacth $=20$ $\operatorname{dist}<3 * \min$ dist 


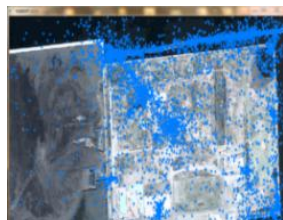

Ransacth $=1$

dist $<3 *$ min dist

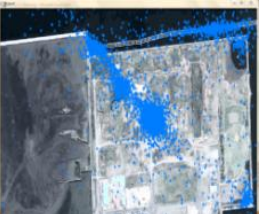

Ransacth $=50$

dist $<3 *$ min dist

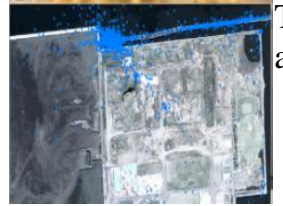

Ransacth $=90$

dist $<3 *$ min dist

Fig. 19. Localization output at different threshold values

Fig. 19 shows the variation in localization at different values of the threshold. As the value of RANSAC is increased the number of mismatches is drastically reduced along with correct interest points. Hence in my project an RANSAC threshold is kept at a value of 50.

\section{- Use of Histogram Equalization}

When the histogram equalization was included the points are less scattered or mismatched (i.e the keypoints localized tends to be more accurate). Hence, the accuracy was comparatively increased considering the image on which histogram equalization was not carried out (were points tend to be less detected). The comparison is shown in Fig. 21 and 22.

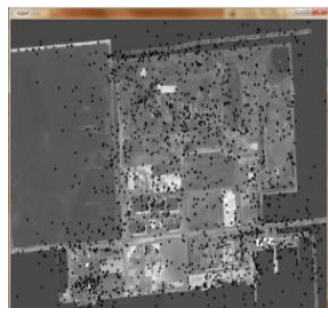

(a)

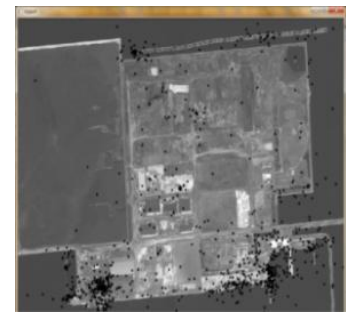

(b)

Fig. 20. Localization using (a) SURF and FLANN algorithm (b) SURF, FLANN and RANSAC algorithm

When histogram equalization is applied to the image, for contrast equalization, the localized points are less scattered and mismatched when compared with a localized output without histogram equalization.

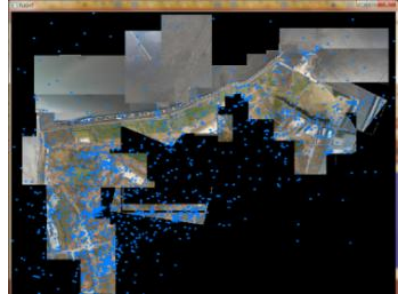

Fig. 21. Matching without histogram equalization

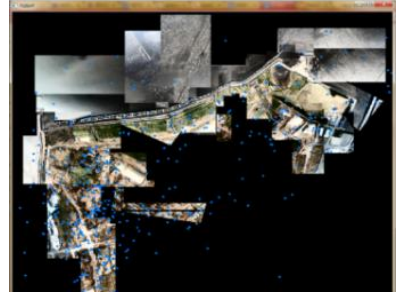

Fig. 22. Matching with histogram equalization

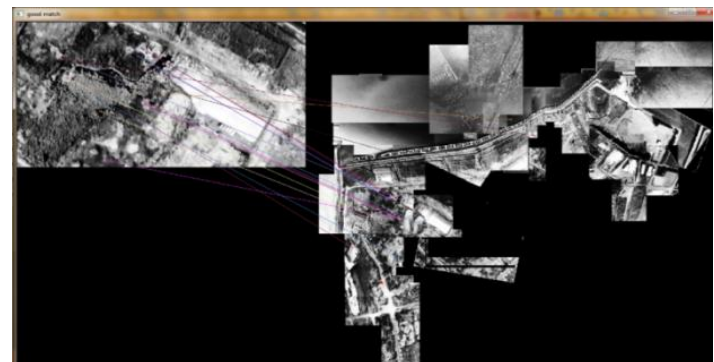

Fig. 23. Contrast enhancement based mapping

The above figure, Fig. 23 shows the matching performed after contrast enhancement.

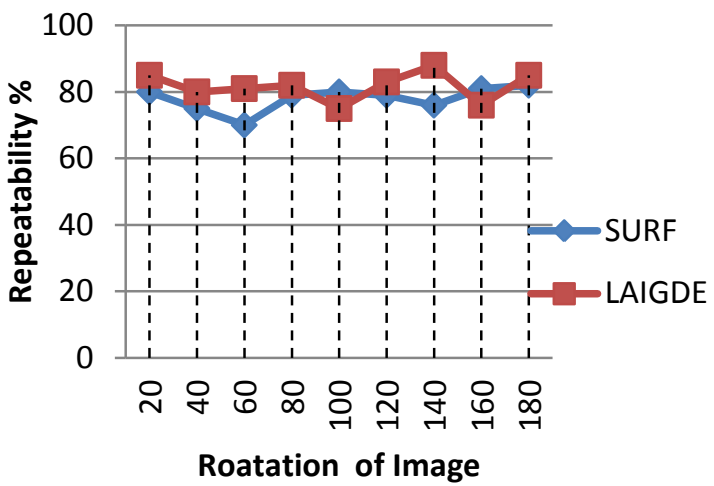

Fig.24.Repeatability of LAIGDE Vs SURF

From Fig. 24 the repeatability score for varying angle of rotation of the image in LAIGDE algorithm is found to be efficient in comparison to SURF approach.

\section{CONCLUSION}

In this project, an efficient way to localize a suborbital vehicle was presents. The program was developed in CPP in visual studio 2012. The fewer mismatches spotted in surf algorithm was eliminated using the histogram equalization of the images. Hence, in a GPS-denied environment, employment of such an algorithm can help in localization of unmanned aerial vehicle efficiently and more accurately compared to the traditional surf approach. Integration of odometry values to find the exact location with great accuracy will be an interesting future research.

\section{ACKNOWLEDGMENT}

I would like to thank all the faculties and all my teachers (all my school teachers and college lecturers/professors) and supporters (my parents and friends) who came to my life and lead me towards a research path. I would especially like to thank my parents, SRM University and my faculties and friends from KIT Japan for their support and guidance. I thank SRM University and my faculties for providing me with a research atmosphere and for their constant support and guidance. I would like to thank my faculties and lab members from Space Systems Laboratory, KIT, Japan for their constant support and guidance throughout my coursework and my stay.

\section{REFERENCES}

[1] Chandrakala M., Amssaveni R.(2013) "Classification of Remote Sensing Image Areas Using SURF Features and Latent DirichletAllocation," International Journal of Advanced Research in Computer Science and Software Engineering.

[2] F. Chenavier and J. Crowley, "Position estimation for a mobile robot using vision and odometry", Proceedings 1992 IEEE International Conference on Robotics and Automation.

[3] FarajAlhwarin, DanijelaRistic-Durrant, Axel Graser. (2010) "Speeded Up Image Matching SPLIT and Extended SIFT Features," International Journal on Computer Vision Theory and Applications. 
4] Luo Juan, OubongGwun."A Comparison of SIFT, PCA-SIFT and SURF," International Journal on Image Processing Volume 930, Issue (4.

[5] C. Wang, T. Wang, J. Liang, Y. Zhang and Y. Zhou, "Bearing-only Visual SLAM for Small Unmanned Aerial Vehicles in GPS-denied Environments", International Journal of Automation and Computing, vol. 10, no. 5, pp. 387-396, 2013.

[6] Yan GONG, Hong XIE, Lei YU. (2013) "Image Mosaic Based on SURF Feature Match and Similarity Transformation," Journal of Computational Information System, pp 2-8.

7] Velmurugan K., SanthoshBabooS.Dr.Lt. (2011) "Content -based Image Retrieval Using SURF and Color Moments," Global Journal of Computer Science and Technology, Volume 11, Issue10, Version 1.0, pp.2-4

[8] M. Cummins and P. Newman, "FAB-MAP: Probabilistic Localization and Mapping in the Space of Appearance", The International Journal of Robotics Research, vol. 27, no. 6, pp. 647-665, 2008.

[9] David G. Lowe. (2004)"Distinctive Image Features from ScaleInvariant Key-points," International Journal of Computer Vision.

[10] Tuhin Shukla, Nishchol Mishra, Sanjeev Sharma. (2013) "Automatic Image Annotation Using SURF Features," International Journal of Computer Application" Volume 68-No.4.

[11] Jan Elseberg, StephaneMagnenat, Roland Siegwart, Andreas Nuchter.(2012)"Comparison of Nearest-Neighbor-Search Strategies and Implementation for efficient shape registration," Journal of Software Engineering For Robotics, pp.4-11.

12] Xiusshan NIE, Ju LIU, Jiande SUN, Huawei ZHAO. (2011) "KeyFrame Based Robust Video Hashing Using Isometric Feature Mapping," Journal of Computational Systems.

[13] G. Conte and P. Doherty, "Vision-Based Unmanned Aerial Vehicle Navigation Using Geo-Referenced Information", EURASIP Journal on Advances in Signal Processing, vol. 2009, pp. 1-19, 2009.

[14] Xing Xiong, Byung-Jae Choi. (2013)"Improvement on Image Restoration for Relative Self-Localization Estimation," International Journal of Multimedia and Ubiquitous Engineering.

[15] R. Bouchiha and K. Besbes, "Automatic Remote-sensing Image Registration Using SURF", IJCTE, pp. 88-92, 2013.

[16] V. A, D. Hebbar, V. Shekhar, K. Murthy and S. Natarajan, "Two Novel Detector-Descriptor Based Approaches for Face Recognition Using SIFT and SURF", Procedia Computer Science, vol. 70, pp. 185-197, 2015

[17] M. Dawood, C. Cappelle, M. El Najjar, M. Khalil, B. El Hassan, D. Pomorski and J. Peng, "Virtual 3D city model as a priori information source for vehicle localization system", Transportation Research Part C: Emerging Technologies, vol. 63, pp. 1-22, 2016.

[18] J. Xingteng, W. Xuan and D. Zhe, "Image matching method based on improved SURF algorithm", 2015 IEEE International Conference on Computer and Communications (ICCC), 2015.

[19] E. Garcia-Fidalgo and A. Ortiz, "Vision-based topological mapping and localization methods: A survey", Robotics and Autonomous Systems, vol. 64, pp. 1-20, 2015.

[20] Su Juan, Xu Qingsong and Zhu Jinghua, "A scene matching algorithm based on SURF feature", 2010 International Conference on Image Analysis and Signal Processing, 2010.

[21] E. Fernández-Moral, P. Rives, V. Arévalo and J. González-Jiménez, "Scene structure registration for localization and mapping", Robotics and Autonomous Systems, vol. 75,pp. 649-660, 2016.

\section{BIOGRAPHY}

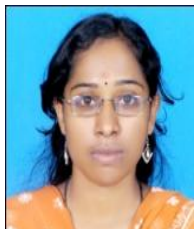

M. Jisha has completed her B.E (ECEElectronics and Communication Engineering) from Narayana Guru College of Engineering, Kanyakumari (2008-2012) affiliated to Anna University, Chennai. She has also completed her M.Tech (Robotics) from SRM University, Chennai, India (20132014). During her M.Tech, she was selected as a special research student, from SRM University, to study in Kyushu Institute of Technology, Tobata, Japan for six months. She has handful experience working in the field of image processing and mobile ad hoc networks and mobile communication. Her research interests include wireless energy efficient and reliable networks, WSN, MANETs, Robotics, Image processing and Digital Electronics, as well as next generation technologies. She is currently working as a Research Associate in Grian Technologies Pvt. Ltd, Chennai. 